\title{
A new nomogram from the SEER database for predicting the prognosis of gallbladder cancer patients after surgery
}

\author{
Zunqiang Xiao" ${ }^{1 \#}$, Zhan Shi ${ }^{1 \#}$, Linjun $\mathrm{Hu}^{2}$, Yuling $\mathrm{Gao}^{3}$, Junjun $\mathrm{Zhao}^{4}$, Yang $\mathrm{Liu}^{2}$, Qiuran $\mathrm{Xu}^{5}$, \\ Dongsheng Huang ${ }^{5}$
}

${ }^{1}$ The Second Clinical Medical College of Zhejiang Chinese Medical University, Hangzhou 310014, China; ${ }^{2}$ The Medical College of Qingdao University, Qingdao 266071, China; ${ }^{3}$ Department of Genetic Laboratory, Shaoxing Women and Children Hospital, Shaoxing 312030, China; ${ }^{4}$ Graduate Department, Bengbu Medical College, Bengbu 233030, China; ${ }^{5}$ The Key Laboratory of Tumor Molecular Diagnosis and Individualized Medicine of Zhejiang Province, Zhejiang Provincial People's Hospital (People's Hospital of Hangzhou Medical College), Hangzhou 310014, China Contributions: (I) Conception and design: Z Xiao, Z Shi; (II) Administrative support: Q Xu, D Huang; (III) Provision of study materials or patients: L Hu, Y Gao; (IV) Collection and assembly of data: J Zhao, Yang Liu; (V) Data analysis and interpretation: Z Xiao, Z Shi; (VI) Manuscript writing: All authors; (VII) Final approval of manuscript: All authors.

\#These authors contributed equally to this work as co-first authors.

Correspondence to: Qiuran Xu, MD, PHD; Dongsheng Huang, MD, PhD. Key Laboratory of Tumor Molecular Diagnosis and Individualized Medicine of Zhejiang Province, Zhejiang Provincial People's Hospital (People’s Hospital of Hangzhou Medical College), Hangzhou 310014 , China. Email: windway626@sina.com; dshuang@zju.edu.cn.

Background: To study the prognostic significance in gallbladder cancer (GBC) patients of the four $\mathrm{N}$ stage methods of log odds of positive lymph nodes (LODDS), lymph node ratio (LNR), and N stage in the 7th and $8^{\text {th }}$ editions of the American Joint Committee on Cancer (AJCC), and to establish a prognostic model of GBC based on LODDS.

Methods: Data of 1,321 patients with GBC who underwent surgical resection of lymph nodes from 2010 to 2014 were collected from the Surveillance, Epidemiology, and End Results (SEER) database. We then randomly divided these data into a training set $(n=925)$ and a validation set $(n=396)$. C-index, Akaike information criterion (AIC), and area under the curve (AUC) were calculated to evaluate the accuracy of LODDS, LNR, and N stage in the $7^{\text {th }}$ and $8^{\text {th }}$ editions of the AJCC. Cox multivariate analysis was performed to determine whether LODDS was an independent prognostic factor, and a nomogram model was established. C-index was used to evaluate the accuracy of the nomogram. A receiver operating characteristic (ROC) curve was drawn and the area under the AUC was calculated to evaluate the accuracy of the nomogram in predicting patients' 1-, 3-, and 5-year overall survival (OS).

Results: Univariate analysis showed that the four methods were all correlated with OS. Through C-index, AIC and AUC, We found that LODDS had the best accuracy of the four methods. C-index and AUC analysis revealed that the nomogram based on LODDS had excellent prognostic ability. All the results were verified in the validation set.

Conclusions: LODDS is an independent prognostic factor for GBC patients, and it is the best $\mathrm{N}$ stage in the SEER database. This new nomogram-containing LODDS system is a great model to predict the prognosis of GBC patients.

Keywords: Gallbladder cancer (GBC); prognostic model; nomogram; LODDS

Submitted Nov 04, 2019. Accepted for publication Nov 21, 2019.

doi: 10.21037/atm.2019.11.112

View this article at: http://dx.doi.org/10.21037/atm.2019.11.112 


\section{Introduction}

Gallbladder carcinoma is the most common malignant tumor whose incidence ranks sixth in digestive system tumor (1). Compared with other malignant bile duct tumors, gallbladder cancer (GBC) has a poorer prognosis, and it is difficult to diagnose it in the early stage $(2,3)$. Currently, cholecystectomy is still the main treatment to improve the prognosis of GBC. One of the important factors affecting the curative effect of radical cholecystectomy for GBC is lymph node metastasis, as it is the main channel of metastasis for GBC with a rate in $\mathrm{T} 3$ and $\mathrm{T} 4$ patients of 60-80\% (4-7). Consequently, intraoperative lymph node dissection and the precise preoperative staging of lymph node metastasis based on the results of intraoperative lymph node dissection is helpful for evaluating prognosis and providing individualized treatment.

In the $7^{\text {th }}$ edition of the American Joint Committee on Cancer (AJCC) tumor TNM staging system (8), the N stage of gallbladder carcinoma is divided into $\mathrm{N} 1$ and $\mathrm{N} 2$ according to the location of lymph node metastasis, while the 8 th edition of the cancer staging system is based on the number of metastatic lymph nodes (NMLN). However, there are still few high-level evidence-based medical data available to determine whether the $8^{\text {th }}$ edition's $\mathrm{N}$ staging is more instructive than other methods for prognosis. Furthermore, in view of the different resected lymph nodes (RLNS) and lymph nodes dissection areas, it is difficult to evaluate the prognosis of GBC patients only by NMLN. Some scholars believe that lymph node ratio (LNR) is more accurate than $\mathrm{N}$ staging in predicting the survival of patients with GBC after operation (9-12). The LNR methods include the log odds of metastatic lymph node (LODDS) and LNR. LODDS is defined as the ratio of the number of positive lymph nodes (PLNS) to the number of negative nodes, while LNR is defined as the ratio of PLNS to RLNS. Numerous studies on pancreatic cancer (13), oral cancer (14), and colorectal cancer (15) have shown that LNR and LODDS are good prognostic indicators, and they been accepted as predictors of prognosis. Nomograms can integrate multiple prediction indexes based on multivariate regression analysis, and then assign scores to each factor according to a regression coefficient to predict the probability of an event by adding up the scores. As a new, intuitive diagram which can predict patient prognosis directly, the nomogram has been used for evaluating the prognosis of cancer patients (16). In this study, univariate analysis was used to determine which of the four $\mathrm{N}$ stage methods (LODDS, LNR, and N stage in the 7th and 8th editions of the AJCC) could be a potential prognostic factor. We then compared the accuracy of the four $\mathrm{N}$ stage methods and screened out the best $\mathrm{N}$ stage method, which was the LODDS system. Cox multivariate analysis was used to determine whether LODDS was an independent prognostic factor, and a nomogram containing LODDS was established. Finally, we evaluated the accuracy of this nomogram and verified it in a validation set.

\section{Methods}

We collected data from the Surveillance, Epidemiology, and End Results (SEER) database of the American Cancer Institute (http://seer.cancer.gov/) on GBC patients who underwent lymphadenectomy from 2010 to 2014. The selection criteria were as follows: (I) all data contained ICD-O-3 histopathological classification, and all patients were diagnosed histopathologically; (II) all data had detailed TNM stage information, and pathological stage was classified by the 7th Edition [2010] TNM of the United States Joint Cancer Commission (AJCC). The exclusion criteria were as follows: (I) unknown age; (II) unknown TMN stage; (III) unknown tumor type; (IV) unknown tumor size; (V) unknown grade stage; (VI) no lymph node resection or no lymph node information. After analyzing the TNM stage criteria of GBC in the $7^{\text {th }}$ and $8^{\text {th }}$ edition of AJCC, we found that in the $8^{\text {th }}$ edition, only the staging criteria of $\mathrm{N}$ stage changed compared with the $7^{\text {th }}$ edition. Thus, we could obtain the data of TNM stage in $8^{\text {th }}$ edition for these patients by transforming the data of NMLN to N stage in the $8^{\text {th }}$ edition. Finally, the data of 1,321 patients with GBC were included in this study. The patients were divided into two groups (the training set and validation set) using R software Caret package. In this study, all histological types, except for adenomas and adenocarcinomas, were described as "other" (Figure 1).

\section{Statistical analysis}

Univariate analysis and multivariate analysis were used to evaluate the prognosis and screen out some potential risk factors of GBC patients. Harrell C-index and the Akaike information criterion (AIC) were used to analyze the accuracy of LODDS, LNR, N stage in $7^{\text {th }}$ and $8^{\text {th }}$ editions of the AJCC. Moreover, a receiver operating characteristic (ROC) curve was drawn and area under the curve (AUC) was calculated to evaluate the accuracy of LODDS, LNR, 


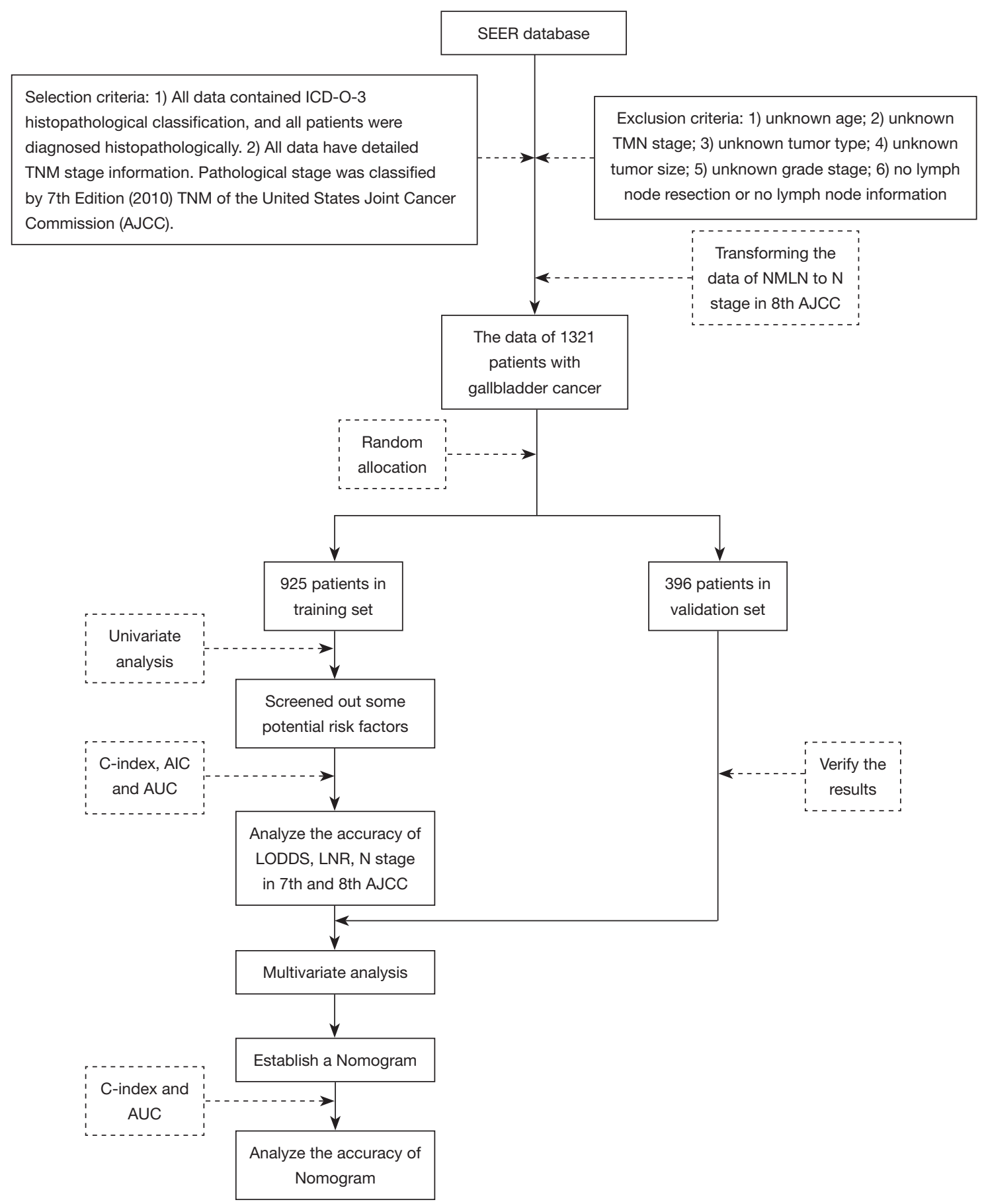

Figure 1 Graphical abstract.

and $\mathrm{N}$ stage in the $7^{\text {th }}$ and $8^{\text {th }}$ editions of the AJCC for overall survival (OS) of GBC patients 1 and 3 years after operation. Finally, Harrell C-index and AUC were used to analyze the accuracy of the nomogram. R software was used for all statistical analyses. A $\mathrm{P}$ value $<0.05$ was considered statistically significant.

\section{The LODDS system}

The LODDS is defined as $\log$ [(the number of PLNs 
Page 4 of 12

$+0.05) /($ the number of negative nodes +0.05$)]$. As the LODDS range in our research was ( -2.903 to 2.417$)$, the LODDS system was grouped into four classifications with an interval of 1.5: LODDS1 (LODDS $\leq-1.5$ ), LODDS2 $(-1.5 \leq$ LODDS $<0)$, LODDS3 $(0 \leq$ LODDS $<1.5)$ and LODDS4 (LODDS $\geq 1.5$ ).

\section{LNR}

LNR is defined as (the ratio of PLNS/RLNS). The LNR was grouped into four classifications with an interval 0.25: LNR1 $(0 \leq \mathrm{LNR}<0.25)$, LNR2 $(0.25 \leq \mathrm{LNR}<0.5)$, LNR3 $(0.5 \leq \mathrm{LNR}<0.75), \mathrm{LNR} 4(0.75 \leq \mathrm{LNR} \leq 1)$.

\section{Results}

\section{Patients' characteristics}

A total of 925 patients were included in the training set, and 396 patients were included in the validation set. The clinical data of the two sets are shown in Table 1. There was no significant difference in age, sex, tumor size, grade, histology, T stage ( $8^{\text {th }}$ AJCC), M stage ( $8^{\text {th }}$ AJCC), N stage
Xiao et al. A new nomogram for predicting the prognosis of GBC

( $7^{\text {th }}$ AJCC and $8^{\text {th }}$ AJCC), LODDS system, RLNS, LNR, and PLNRS between the training set and the verification set $(\mathrm{P}<0.05)$.

\section{Independent prognostic factors of $O S$ in the training set}

Univariate analysis showed that age, T stage $\left(8^{\text {th }}\right.$ AJCC), $M$ stage $\left(8^{\text {th }}\right.$ AJCC), $\mathrm{N}$ stage $\left(7^{\text {th }}\right.$ AJCC and $8^{\text {th }}$ AJCC), LODDS system, LNR, tumor size, grade, and histology were correlated with $\mathrm{OS}(\mathrm{P}<0.05$, Table 2, Figure 2). The predicted $\mathrm{C}$-index, AIC, and AUC values of the ROC predicted the 1- and 3-year OS for LODDS to be 0.649 , $5,183,0.674$, and 0.703 , respectively. The predicted C-index, AIC, and AUC values of the ROC predicted the 1 - and 3 -year OS for $\mathrm{N}$ stage in the $7^{\text {th }}$ edition of the AJCC to be $0.627,5,190,0.650$, and 0.688 , respectively. The predicted C-index, AIC, and AUC values of the ROC predicted the 1- and 3-year OS for $\mathrm{N}$ stage in $8^{\text {th }}$ edition of the AJCC to be $0.627,5,191,0.650$, and 0.687 , respectively. The predicted C-index, AIC, and AUC values of the ROC predicted the 1- and 3-year OS for LNR to be $0.634,5,190$, 0.662 , and 0.680 , respectively (Table 3). Multivariate Cox analysis showed that age, $T$ stage, $M$ stage, LODDS system,

Table 1 Demographic and clinicopathological characteristics of patients with gallbladder cancer between 2010 and 2014 in the SEER database

\begin{tabular}{|c|c|c|c|c|}
\hline Demographic or characteristics & All subjects $(\mathrm{N}=1,321)$ & Training set $(\mathrm{N}=925)$ & Validation set $(\mathrm{N}=396)$ & $P$ value \\
\hline Age at diagnosis (year) & & & & 0.409 \\
\hline $30-50$ & 90 & 64 & 26 & \\
\hline $50-70$ & 625 & 428 & 197 & \\
\hline$\geq 70$ & 606 & 433 & 173 & \\
\hline Sex & & & & 0.267 \\
\hline Female & 919 & 635 & 284 & \\
\hline Male & 402 & 290 & 112 & \\
\hline $\mathrm{T} 8^{\text {th }}$ stage & & & & 0.472 \\
\hline $\mathrm{T} 1$ & 159 & 119 & 40 & \\
\hline T2 & 658 & 451 & 207 & \\
\hline T3 & 465 & 328 & 137 & \\
\hline $\mathrm{T} 4$ & 39 & 27 & 12 & \\
\hline$M 8^{\text {th }}$ stage & & & & 0.087 \\
\hline Mo & 1,143 & 790 & 353 & \\
\hline M1 & 178 & 135 & 43 & \\
\hline
\end{tabular}

Table 1 (continued) 
Table 1 (continued)

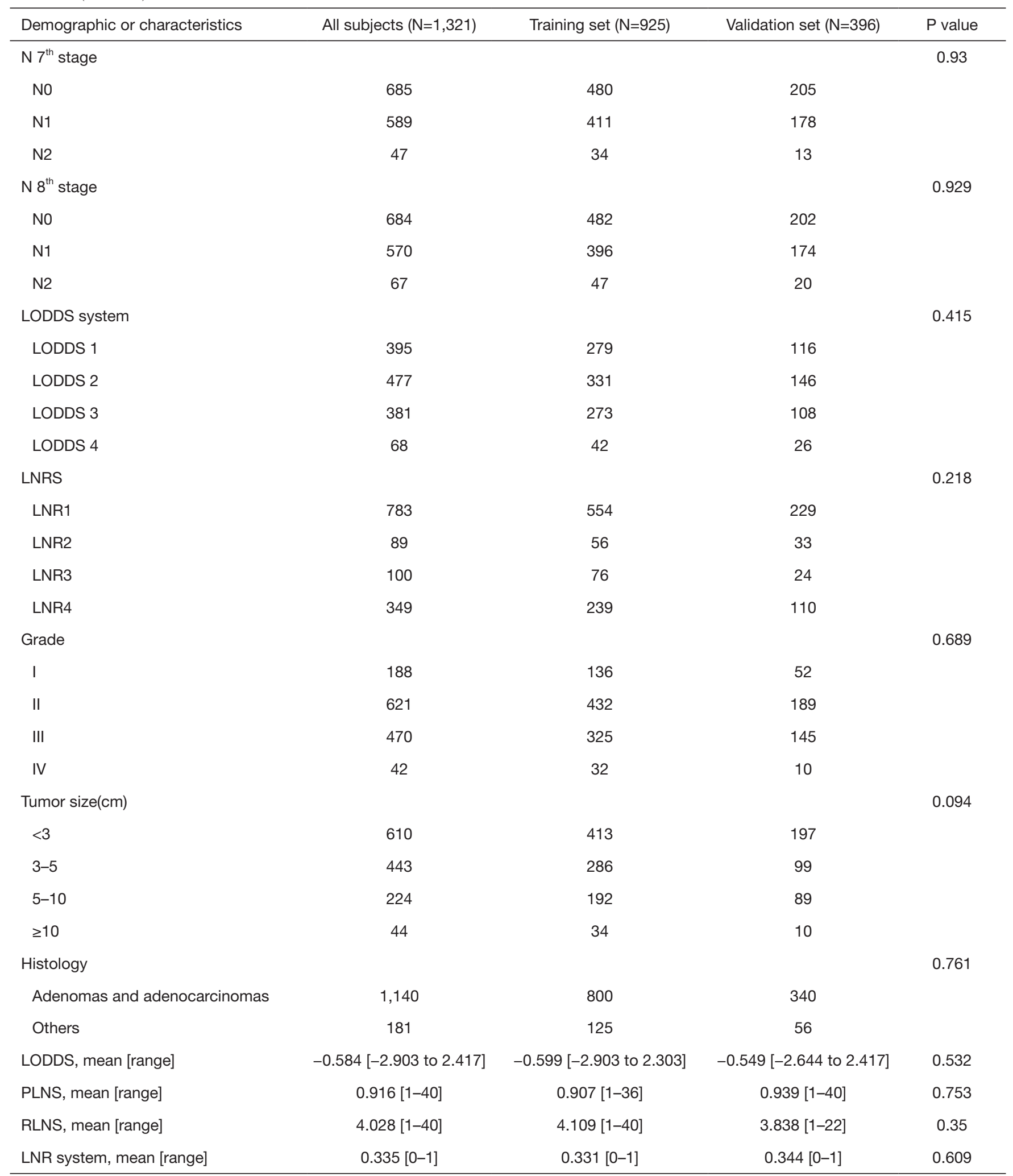

T, N, M stage come from AJCC. SEER, Surveillance, Epidemiology, and End Results; LNR, lymph node ratio; AJCC, American Joint Committee on Cancer. 
Table 2 Univariate analysis in the training set

\begin{tabular}{lll}
\hline \multirow{2}{*}{ Variables } & \multicolumn{2}{c}{ Univariate analysis } \\
\cline { 2 - 3 } & $\mathrm{HR}(95 \% \mathrm{Cl})$ & P value
\end{tabular}

Age at diagnosis

$30-50$

Reference

$50-70$

$\geq 70$

$1.291(0.836-1.993)$

$1.966(1.279-3.020)$

$T 8^{\text {th }}$ stage

T1

T2

T3

T4

$N 7^{\text {th }}$ stage

NO

N1

N2

$\mathrm{N} 8^{\text {th }}$ stage

NO

N1

N2

$M 8^{\text {th }}$ stage

Mo

M1

LNR system

LNR1

LNR2

LNR3

LNR4

Grade

I

II

III

IV

Tumor size (cm)

$<3$

3-5

5-10

$\geq 10$

Table 2 (continued)
0.248

0.002

Table 2 (continued)

\begin{tabular}{|c|c|c|}
\hline \multirow{2}{*}{ Variables } & \multicolumn{2}{|c|}{ Univariate analysis } \\
\hline & $\mathrm{HR}(95 \% \mathrm{Cl})$ & $P$ value \\
\hline \multicolumn{3}{|l|}{ Histology } \\
\hline $\begin{array}{l}\text { Adenomas and } \\
\text { adenocarcinomas }\end{array}$ & Reference & \\
\hline Others & $1.646(1.279-2.119)$ & $<0.001$ \\
\hline \multicolumn{3}{|l|}{ LODDS system } \\
\hline LODDS 1 & Reference & \\
\hline LODDS 2 & 1.975 (1.495-2.609) & $<0.001$ \\
\hline LODDS 3 & 3.793 (2.885-4.989) & $<0.001$ \\
\hline LODDS 4 & $4.986(3.257-4.633)$ & $<0.001$ \\
\hline \multicolumn{3}{|l|}{ Sex } \\
\hline Female & Reference & \\
\hline Male & $1.020(0.830-1.253)$ & 0.848 \\
\hline
\end{tabular}

$<0.001$

$<0.001$

tumor size, and grade were independent prognostic factors $(\mathrm{P}<0.05$, Table 4).

\section{Prognostic nomogram for $O S$}

Independent risk factors, including age, tumor size, grade, $T$ stage, $M$ stage and LODDS system were selected to draw the OS prognostic nomogram (Figure 3). The calibration plot for the probability of survival at 1,3 , and 5 years after surgery showed an optimal agreement between the prediction by nomogram and actual observation in the training set (Figure $4 A, B, C$ ) and in the validation set (Figure $4 D, E, F$ ). The predicted C-index of the nomogram in the training set was 0.752 (95\% CI, 0.768 to 0.720 ), and the AUC values of the ROC predicted the 1-, 3-, and 5 -year OS of the nomogram to be $0.804,0.820$, and 0.804 , respectively (Figure $5 A, B, C$ ). The predicted C-index of the nomogram in the validation set was 0.752 (95\% CI, 0.768 to 0.720 ), and the AUC values of the ROC predicted the 1-, 3 -, and 5-year OS of the nomogram to be $0.799,0.795$, and 0.824 , respectively (Figure 5D,E,F).

\section{Discussion}

Lymph node metastasis is an important prognostic factor in patients with GBC after radical operation (17-19). Its effect 
A

$\mathrm{N} 7^{\text {th }}$ stage

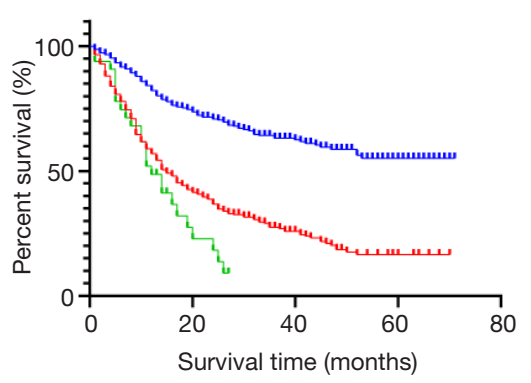

C

LODDS system

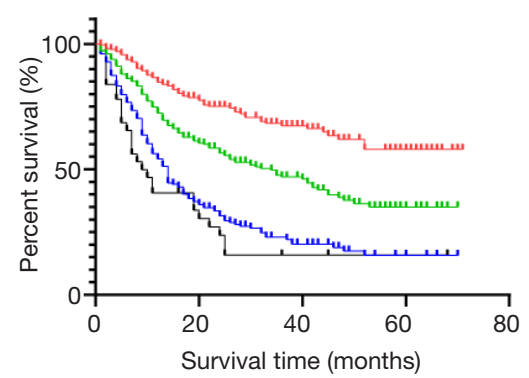

B

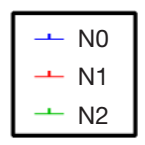

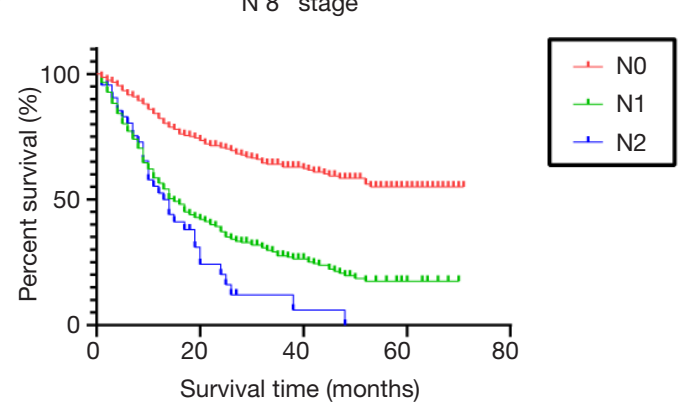

D

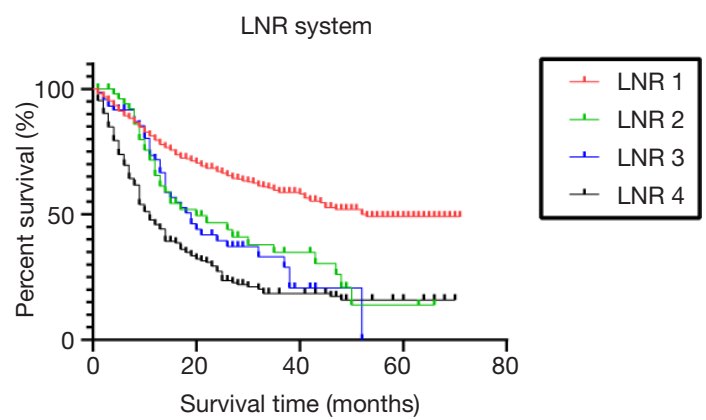

Figure 2 Kaplan-Meier OS curve stratified by patient characteristics in the training set: (A) $\mathrm{N} 7^{\text {th }}$ stage; (B) $\mathrm{N} 8^{\text {th }}$ stage; (C) LODDS system; (D) LNR system; statistical analysis of each factor, $\mathrm{P}<0.01$. OS, overall survival; LNR, lymph node ratio.

Table 3 Prognostic efficiency of different lymph node staging systems

\begin{tabular}{|c|c|c|c|c|}
\hline \multirow{2}{*}{ System } & \multirow{2}{*}{ C-index } & \multirow{2}{*}{$\mathrm{AIC}$} & \multicolumn{2}{|c|}{ AUC } \\
\hline & & & 1 year survival & 3-year survival \\
\hline LODDS system & 0.649 & 5,183 & 0.674 & 0.703 \\
\hline $\mathrm{N} 7^{\text {th }}$ stage & 0.627 & 5,190 & 0.650 & 0.688 \\
\hline$N 8^{\text {th }}$ stage & 0.627 & 5,191 & 0.650 & 0.687 \\
\hline
\end{tabular}

$\mathrm{N}$ stage comes from AJCC. AIC, Akaike information criterion; LNR, lymph node ratio; AUC, area under the curve; AJCC, American Joint Committee on Cancer.

on prognosis is more substantial than $\mathrm{T}$ stage in advanced gallbladder carcinoma patients with localized lesions (20). In view of its importance in the diagnosis and treatment of GBC, there are many stage criteria for lymph node metastasis $(12,21)$. Although the 7th edition of the AJCC N stage is presently the most widely used stage criteria for lymph node metastasis, if we consider different forms of lymph node stations in the Japanese Society of Biliary Surgery (JSBS) stage system, the prognostic value of the affiliation of some lymph nodes still remains controversial (22). The recently published 8th edition of the AJCC Biliary Malignant Tumor Stage System has made the following modifications to the $\mathrm{N}$ stage formula: the original regional lymph node classification method has been abolished and, except for intrahepatic cholangiocarcinoma, all nodes are classified according to the number of metastatic (positive) lymph nodes which includes 1-3 PLNS for N1, and more than 4 PLNS for N2. However, whether the 8 th edition's $\mathrm{N}$ staging has better clinical practicability and repeatability still needs more clinical verification. 
Table 4 Multivariate analysis in the training set

\begin{tabular}{|c|c|c|}
\hline \multirow{2}{*}{ Variables } & \multicolumn{2}{|c|}{ Multivariate analysis } \\
\hline & HR (95\% Cl) & $P$ value \\
\hline \multicolumn{3}{|l|}{ Age at diagnosis } \\
\hline $30-50$ & Reference & \\
\hline $50-70$ & $1.242(0.802-1.923)$ & 0.331 \\
\hline$\geq 70$ & $2.107(1.363-3.255)$ & $<0.001$ \\
\hline \multicolumn{3}{|l|}{$\mathrm{T}$} \\
\hline $\mathrm{T} 1$ & Reference & \\
\hline $\mathrm{T} 2$ & $1.259(0.831-1.907)$ & 0.277 \\
\hline T3 & $2.606(1.710-3.917)$ & $<0.001$ \\
\hline $\mathrm{T} 4$ & $4.017(2.235-7.218)$ & $<0.001$ \\
\hline \multicolumn{3}{|l|}{ M } \\
\hline MO & Reference & \\
\hline M1 & 2.347 (1.841-2.991) & $<0.001$ \\
\hline \multicolumn{3}{|l|}{ Grade } \\
\hline I & Reference & \\
\hline II & $1.230(0.862-1.753)$ & 0.252 \\
\hline III & $1.382(0.957-1.994)$ & 0.083 \\
\hline IV & $2.294(1.324-3.957)$ & 0.003 \\
\hline \multicolumn{3}{|l|}{ Tumor size (cm) } \\
\hline$<3$ & Reference & \\
\hline $3-5$ & $1.270(1.003-1.607)$ & 0.046 \\
\hline $5-10$ & $1.418(1.102-1.824)$ & 0.006 \\
\hline$\geq 10$ & $2.214(1.384-3.540)$ & $<0.001$ \\
\hline \multicolumn{3}{|l|}{ Histology } \\
\hline $\begin{array}{l}\text { Adenomas and } \\
\text { adenocarcinomas }\end{array}$ & Reference & \\
\hline Others & $1.181(0.899-1.551)$ & 0.231 \\
\hline \multicolumn{3}{|l|}{ LODDS system } \\
\hline LODDS 1 & Reference & \\
\hline LODDS 2 & $1.762(1.330-2.335)$ & $<0.001$ \\
\hline LODDS 3 & $2.602(1.957-3.457)$ & $<0.001$ \\
\hline LODDS 4 & $3.967(2.571-6.121)$ & $<0.001$ \\
\hline
\end{tabular}

T, M stages come from AJCC. AJCC, American Joint Committee on Cancer.
Traditional N-stage is greatly influenced by the number of lymph nodes dissected. In recent years, more attention has been paid to the lymph node dissection of GBC. Lymph node dissection is recommended for GBC in several guidelines. The total number of lymph node dissections recommended by the AJCC 6th edition guideline is more than 3 (23). The total number of lymph node dissections recommended by AJCC's 8 edition guideline is more than $6(1,24) . \mathrm{Wu}(25)$ believes that adequate lymph node dissection should be performed, and at least 15 lymph nodes should be detected to ensure accurate prognosis assessment. However, in practical clinical work, it is difficult to ensure that all patients can have enough lymph node dissection due to the differences of individual patients, surgical methods, and the technical ability of the doctors. In our study, the average number of lymph node dissections was 4.028 , which was lower than the recommended number of lymph node dissections in the AJCC $8^{\text {th }}$ edition. Furthermore, 2 studies reported that the median of the total number of lymph nodes examined (TNLE) in GBC is 1 and 4 respectively $(26,27)$. This would inevitably affect $\mathrm{N}$ stage evaluation, meaning the AJCC $8^{\text {th }}$ edition still has disadvantages in clinical practice. This will inevitably affect the accurate judgement of the number of lymph node metastasis, and then affect the accuracy of $\mathrm{N}$ stage. This phenomenon is called "stage migration". For this reason, the academic eventually proposed that the assessments of lymph node metastasis should be based on NMLN, LNR, and LODDS. Different from the traditional N stage, which only focuses on the location of lymph node metastasis, these three methods have considered the NMLNs, while LNR and LODDS have also considered the number of negative lymph nodes dissected. LNR is the ratio of NMLN to RLNS. Although both NMLN and the extent of lymph node dissection are considered, there are still some limitations in LNR: (I) LNR lacks the ability to distinguish the patients with the same LNR but different RLNS, while LODDS owned. (II) As patients are affected by the extent of lymph node dissection, those with small PLNS might be divided into a higher LNR stage, which would cause statistical bias and lead to unsatisfactory C-index and AUC values based on LNR. This viewpoint has been verified in the studies by Murakami et al. (28), Lee et al. (29), and Sierzega et al. (30). However, the advantages and 


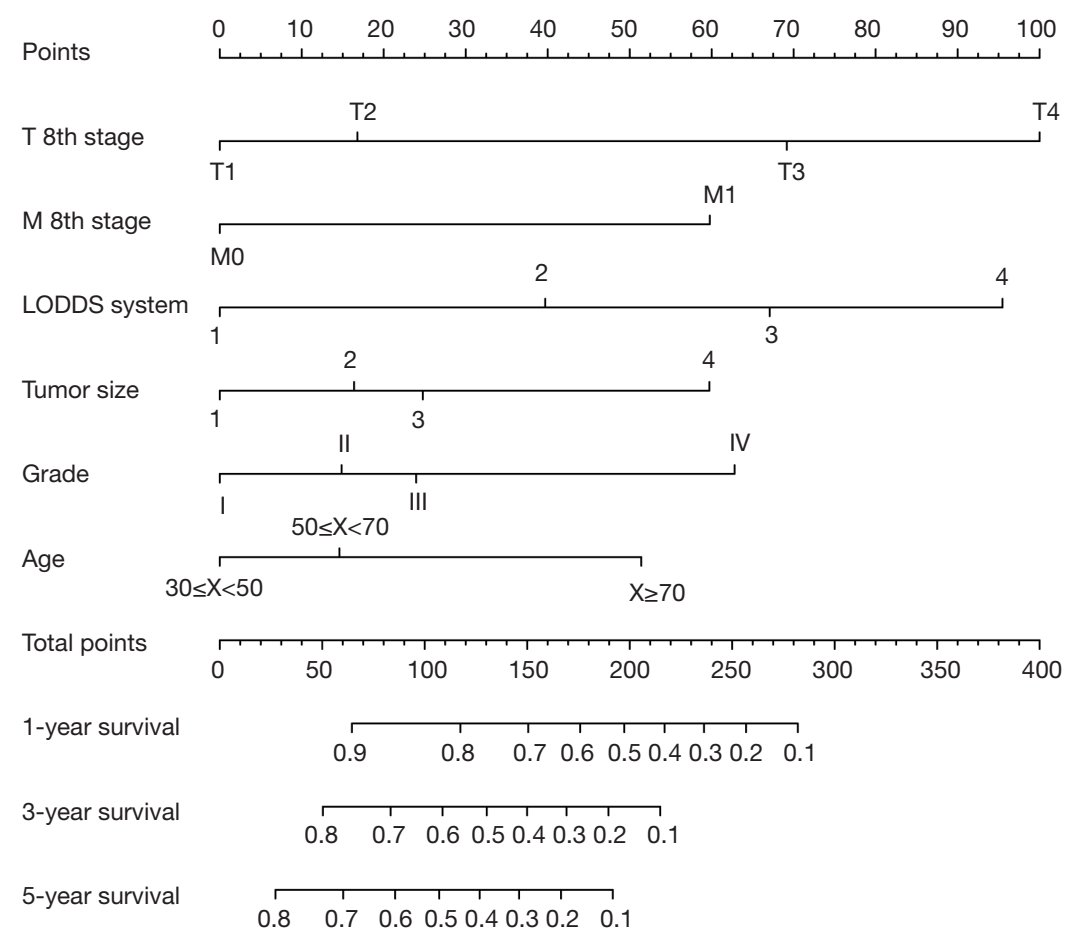

Figure 3 Gallbladder cancer after surgery survival nomogram.

A

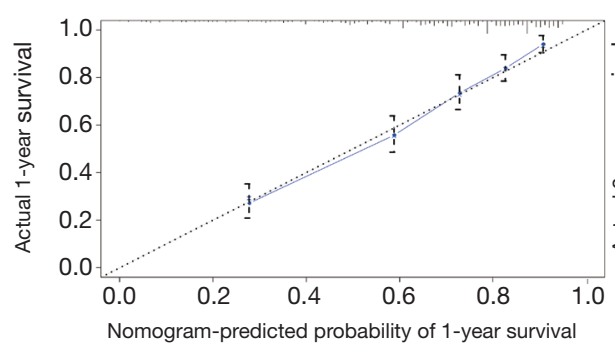

$\mathrm{D}$

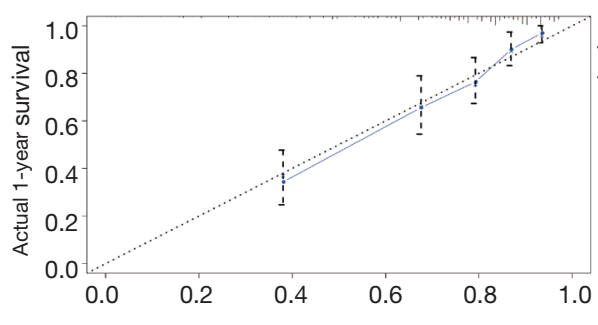

Nomogram-predicted probability of 1-year survival
B

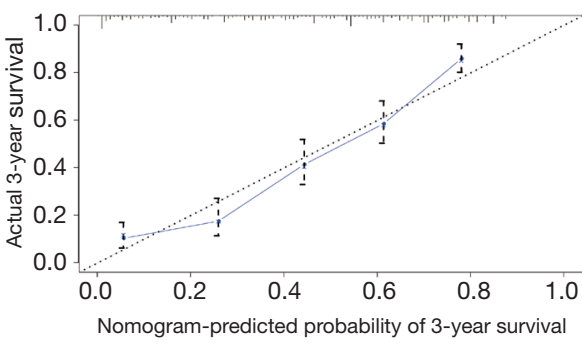

$E$

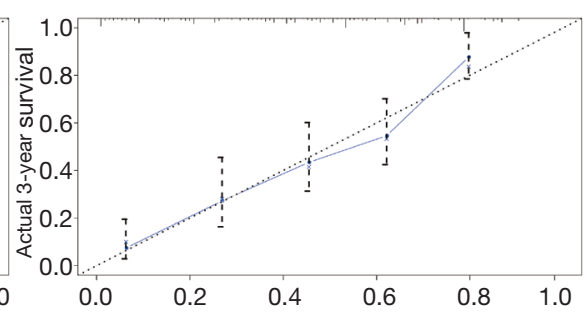

C

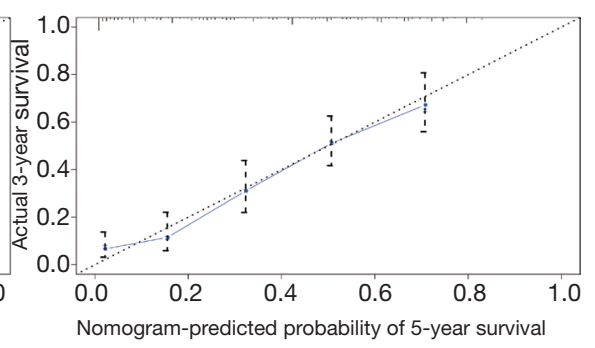

$\mathrm{F}$

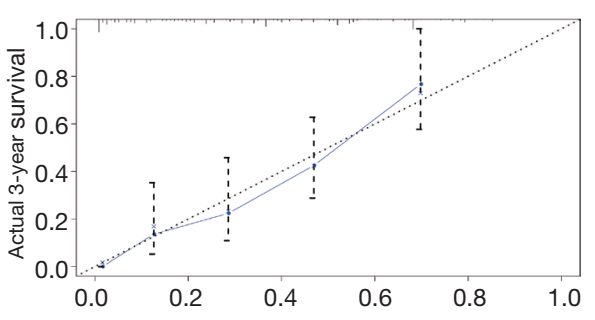

Nomogram-predicted probability of 5-year survival

Figure 4 The calibration curve for predicting patient survival: (A) at 1 year in the training set; (B) at 3 years in the training set; (C) at 5 years in the training set; (D) at 1 year in the validation set; (E) at 3 years in the validation set; (F) at 5 years in the validation set. 
A
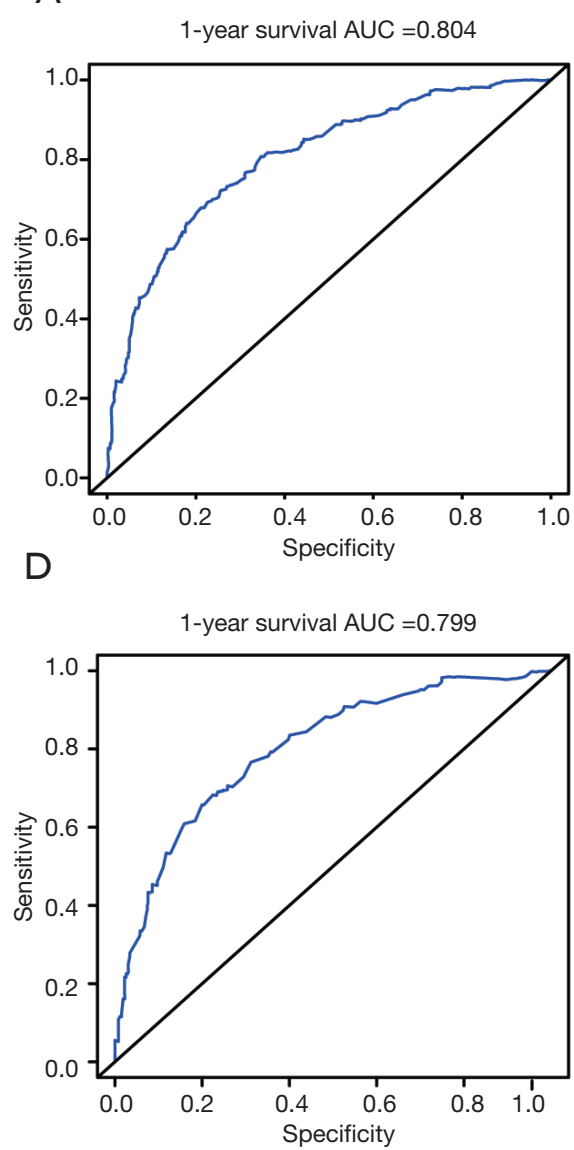

B

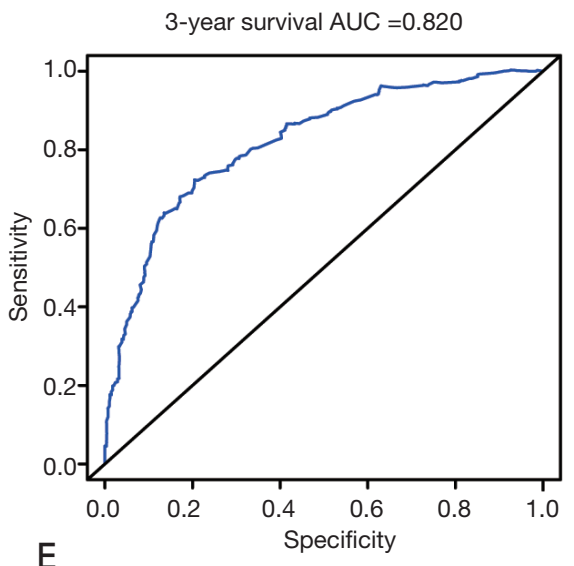

3-year survival AUC $=0.795$

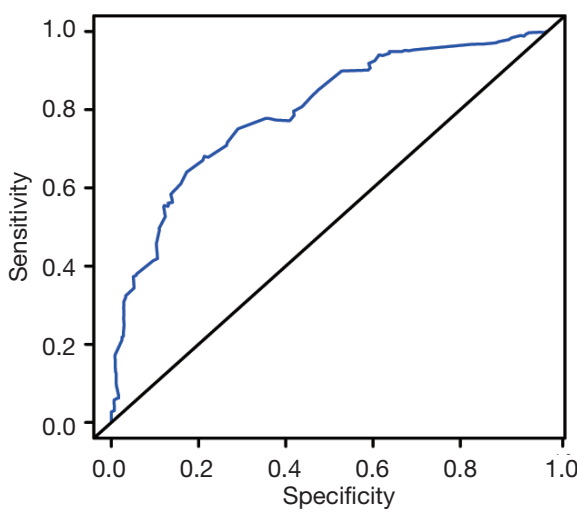

C

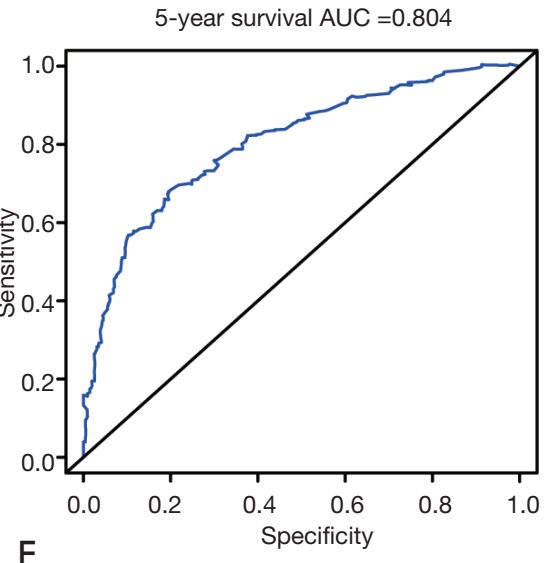

$\mathrm{F}$

5-year survival $A \cup C=0.824$

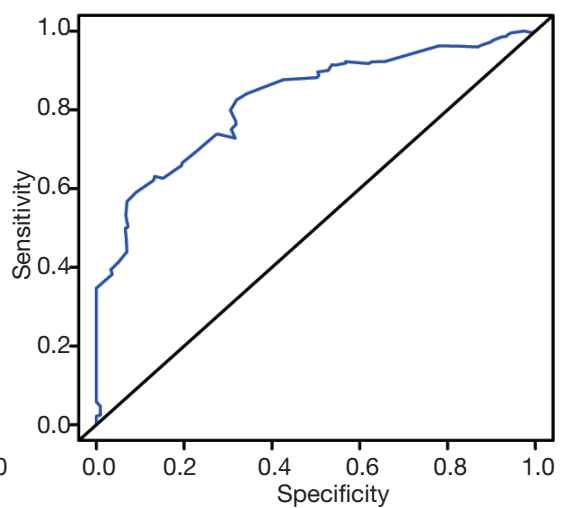

Figure 5 AUC value of ROC predicting: (A) 1-year survival rates of the nomogram in the training set; (B) 3-year survival rates of the nomogram in the training set; (C) 5 -year survival rates of the nomogram in the training set; (D) 1-year survival rates of the nomogram in the validation set; (E) 3-year survival rates of the nomogram in the validation set; (F) 5 -year survival rates of the nomogram in the validation set. AUC, area under the curve; ROC, receiver operating characteristic.

disadvantages of the four methods (N STAGE, PLNRS, LNR, and LODDS) are still uncertain. Amini et al. (26) analyzed 1,124 patients with GBC from the National Cancer Institute (NCI) Surveillance, Epidemiology and Results Database (SEER) database (26), and 214 patients with GBC from a multi-institutional database (27). The results showed that lymph node metastasis stage based on LODDS was the best. Lee et al. analyzed 398 patients with resected $\mathrm{GBC}$, and the result suggested that the LODDS system was helpful in patients with RLN $\geq 6$ (31).

In our study, univariate and multivariate analysis showed that LODDS was an independent risk factor affecting the prognosis of GBC patients. Comparing the C-index, AIC, and AUC of the four methods, we found that LODDS may be a more reliable prognostic factor. Thus, through the
SEER database, we first built a nomogram based on the LODDS system, whose C-Index index and AUC in training set and verification set demonstrated an excellent ability of prognosis. Finally, we verified the accuracy of nomogram in the validation set.

Although LODDS is being gradually more recognized by clinicians and pathologists, there is still no uniform standard for LODDS stratified truncation value. Previous studies have adopted different stratification methods like median and quartile. Different stratification standards lead to different results, which may limit its further application in clinical work. Furthermore, a greater amount of clinical sampling is still needed to verify this nomogram.

In conclusion, LODDS is an independent prognostic factor for GBC patients with lymph node metastasis, and 
it is not affected by the number of lymph nodes sent for examination. It is hopeful that LODDS can provide more accurate guidance in postoperative treatment and prognosis.

\section{Acknowledgments}

The authors would like to thank SEER for open access to the database.

Funding: This study was supported by grants from the National Natural Science Foundation of China (81874049, 81602179), the National Science and Technology Major Project for New Drugs (No. 2017ZX09302003), the Co-construction of Provincial and Department Project (WKJ-ZJ-1919), the Zhejiang Provincial Natural Science Foundation of China (LY19H160036), and the Public Projects of Zhejiang Province (2018C37033).

\section{Footnote}

Conflicts of Interest: The authors have no conflicts of interest to declare.

Ethical Statement: The authors are accountable for all aspects of the work in ensuring that questions related to the accuracy or integrity of any part of the work are appropriately investigated and resolved.

\section{References}

1. Hundal R, Shaffer EA. Gallbladder cancer: epidemiology and outcome. Clin Epidemiol 2014;6:99-109.

2. Sun KK, Shen XJ, Zhao H. An unusual case of cutaneous metastases from gallbladder cancer. Transl Cancer Res 2017;6:1005-8.

3. Hueman MT, Jr CMV, Pawlik TM. Evolving Treatment Strategies for Gallbladder Cancer. Ann Surg Oncol 2009;16:2101-15.

4. Kondo S, Takada T, Miyazaki M, et al. Guidelines for the management of biliary tract and ampullary carcinomas: surgical treatment. J Hepatobiliary Pancreat Surg 2008; 15:41-54.

5. Fong Y, Wagman L, Gonen M, et al. Evidence-based gallbladder cancer staging: changing cancer staging by analysis of data from the National Cancer Database. Ann Surg 2006;243:767-71.

6. Miyakawa S, Ishihara S, Horiguchi A, et al. Biliary tract cancer treatment: 5,584 results from the Biliary Tract Cancer Statistics Registry from 1998 to 2004 in Japan. J
Hepatobiliary Pancreat Surg 2009;16:1-7.

7. Wistuba II, Gazdar AF. Gallbladder cancer: lessons from a rare tumour. Nat Rev Cancer 2004;4:695-706.

8. Edge SB, Compton CC. The American Joint Committee on Cancer: the 7th edition of the AJCC cancer staging manual and the future of TNM. Ann Surg Oncol 2010;17:1471-4.

9. Sakata J, Shirai Y, Wakai T, et al. Number of Positive Lymph Nodes Independently Determines the Prognosis After Resection in Patients with Gallbladder Carcinoma. Ann Surg Oncol 2010;17:1831.

10. Birnbaum DJ, Viganò L, Russolillo N, et al. Lymph node metastases in patients undergoing surgery for a gallbladder cancer. Extension of the lymph node dissection and prognostic value of the lymph node ratio. Ann Surg Oncol 2015;22:811-8.

11. Endo I, Shimada H, Tanabe M, et al. Prognostic significance of the number of positive lymph nodes in gallbladder cancer. J Gastrointest Surg 2006;10:999-1007.

12. Shirai Y, Sakata J, Wakai T, et al. Assessment of lymph node status in gallbladder cancer: location, number, or ratio of positive nodes. World J Surg Oncol 2012;10:87.

13. Ramacciato G, Nigri G, Petrucciani N, et al. Prognostic role of nodal ratio, LODDS, $\mathrm{pN}$ in patients with pancreatic cancer with venous involvement. BMC Surg 2017;17:109.

14. Lee CC, Lin YS, Kang BH, et al. Incorporation of log odds of positive lymph nodes into the AJCC TNM classification improves prediction of survival in oral cancer. Clin Otolaryngol 2017;42:425-32.

15. Arslan NC, Sokmen S, Canda AE, et al. The prognostic impact of the log odds of positive lymph nodes in colon cancer. Colorectal Dis 2014;16:O386-92.

16. Young KA, Efiong E, Dove JT, et al. External Validation of a Survival Nomogram for Non-Small Cell Lung Cancer Using the National Cancer Database. Ann Surg Oncol 2017;24:1459-64.

17. Murakami Y, Uemura K, Sudo T, et al. Prognostic Factors of Patients with Advanced Gallbladder Carcinoma Following Aggressive Surgical Resection. J Gastrointest Surg 2011;15:1007-16.

18. Tsai CY, Yeh CN. Hepatopancreaticoduodenectomy for biliary cancer: operative strategies determine nearzero operative mortality, but tumor biology determines an acceptable long-term outcome. Transl Cancer Res 2017;6:S431-3.

19. Zaydfudim V, Feurer ID, Wright JK, et al. The impact of tumor extent ( $\mathrm{T}$ stage) and lymph node involvement $(\mathrm{N}$ 
stage) on survival after surgical resection for gallbladder adenocarcinoma. HPB 2008;10:420-7.

20. Birnbaum DJ, Viganò L, Ferrero A, et al. Locally advanced gallbladder cancer: which patients benefit from resection? Eur J Surg Oncol 2014;40:1008-15.

21. Oh TG, Chung MJ, Bang S, et al. Comparison of the Sixth and Seventh Editions of the AJCC TNM Classification for Gallbladder Cancer. J Gastrointest Surg 2013;17:925-30.

22. Hwang KY, Yoon YI, Hwang S, et al. Survival analysis following resection of AJCC stage III gallbladder carcinoma based on different combinations of $\mathrm{T}$ and N stages. Korean J Hepatobiliary Pancreat Surg 2015;19:11-6.

23. Greene FL, Page DL, Fleming ID, et al. AJCC Cancer Staging Manual 2002.

24. Amin MB, Greene FL, Edge SB, et al. The Eighth Edition AJCC Cancer Staging Manual: Continuing to build a bridge from a population-based to a more "personalized" approach to cancer staging. CA Cancer J Clin 2017;67:93-9.

25. Wu SG, Li FY, Zhou J, et al. Prognostic value of different lymph node staging methods in esophageal squamous cell carcinoma after esophagectomy. Ann Thorac Surg 2015;99:284-90.

Cite this article as: Xiao Z, Shi Z, Hu L, Gao Y, Zhao J, Liu Y, Xu Q, Huang D. A new nomogram from the SEER database for predicting the prognosis of gallbladder cancer patients after surgery. Ann Transl Med 2019;7(23):738. doi: 10.21037/ atm.2019.11.112
26. Amini N, Spolverato G, Kim Y, et al. Lymph node status after resection for gallbladder adenocarcinoma: prognostic implications of different nodal staging/scoring systems. J Surg Oncol 2015;111:299-305.

27. Amini N, Kim Y, Wilson A, et al. Prognostic Implications of Lymph Node Status for Patients With Gallbladder Cancer: A Multi-Institutional Study. Ann Surg Oncol 2016;23:3016-23.

28. Murakami Y, Uemura K, Sudo T, et al. Number of metastatic lymph nodes, but not lymph node ratio, is an independent prognostic factor after resection of pancreatic carcinoma. J Am Coll Surg 2010;211:196-204.

29. Lee JH, Lee KG, Ha TK, et al. Pattern analysis of lymph node metastasis and the prognostic importance of number of metastatic nodes in ampullary adenocarcinoma. Am Surg 2011;77:322-9.

30. Sierzega M, Nowak K, Kulig J, et al. Lymph node involvement in ampullary cancer: the importance of the number, ratio, and location of metastatic nodes. J Surg Oncol 2009;100:19-24.

31. Lee W, Jeong CY, Kim YH, et al. Validation of the prognostic performance in various nodal staging systems for gallbladder cancer: results of a multicenter study. Langenbecks Arch Surg 2019;404:581-8. 